\title{
Principle of Chirality Hierarchy in Three-Blade Propeller Systems
}

\author{
Shubin Liu \\ Research Computing Center, University of North Carolina, \\ Chapel Hill, North Carolina 27599-3420, USA. \\ Department of Chemistry, University of North Carolina, \\ Chapel Hill, North Carolina 27599-3290, USA. \\ E-mail: shubin@email.unc.edu
}

\begin{abstract}
There are different kinds of molecular chirality, such as zero-dimensional point chirality, onedimensional axial chirality, 2D planar chirality, and 3D chirality. When they coexist in one system, such as in helical structures of proteins and DNA, they form a chirality hierarchy. Earlier, we showed that the chirality propensity of a lower level in a hierarchy is dictated by that of a higher level and henceforth proposed the Principle of Chirality Hierarchy. In this work, we confirm the validity of this principle in the three-blade propeller molecular system. We consider different choices for the three components of a propeller and systematically investigate the dependence of the stereoisomeric energy difference on these choices. Our results show that the preference of the $\mathrm{OD}$ chirality of a functional group in the propeller system is determined by the 1D chirality, and homochirality is also a remarkable feature for this system. We also unveiled that there exists positive cooperativity and electrostatic interactions play the dominant role in determining the stereoisomeric stability difference while the roles for exchange-correlation and steric effects are minor but indispensable. The establishment and confirmation of the Principle of Chirality Hierarchy from this work should find important applications in asymmetric synthesis, macromolecular assembly, and many others.
\end{abstract}


TOC Graphic
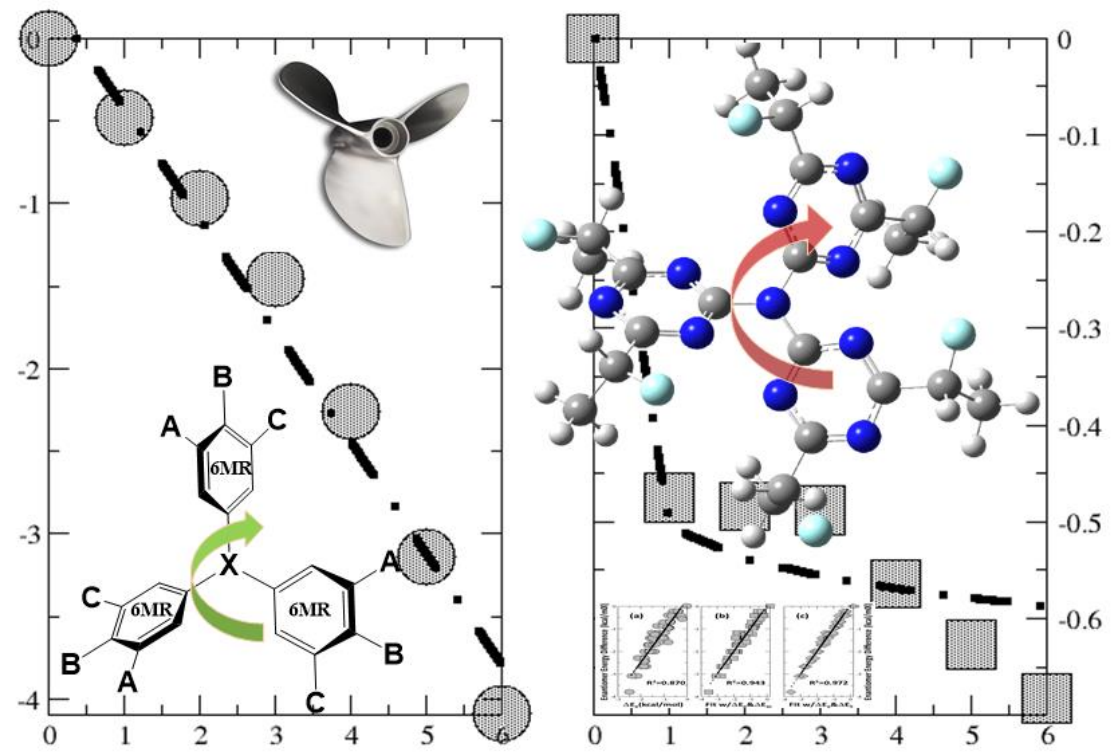

Keywords: Homochirality; Handedness; Propeller; Chirality hierarchy; Density functional theory; Cooperativity; Electrostatic interaction 
Chirality is the geometric property of a molecular system whose mirror image is not superposable to itself. Chiral center as the most common form of molecular chirality is usually called point chirality or OD (zero-dimensional) chirality, which can be labeled by $\mathrm{D} / \mathrm{L}$ based on relative optical activity using Latin words Dexter (right) and Laevus (left), or by its $R$ or $S$ absolute configuration using Latin words Rectus (right) and Sinister (left). ${ }^{1}$ There are other types of chirality in molecules. For example, in axial chirality, a molecule with a helical, propeller, or screw-shaped geometry is held in a spatial arrangement about an axis that is not superposable on its mirror image. This property is also called 1D (one-dimensional) chirality or helicity, expressed by $P$ (plus) or $\Delta$ for right-handedness (counterclockwise), and $M$ (minus) or $\Lambda$ for left-handedness (clockwise). ${ }^{2}$ Similarly, there are planar (2D, two-dimensional) chirality (e.g., in atropisomers), and supramolecular and topological (3D, three-dimensional) chirality reported in the literature. ${ }^{2,3}$ When different kinds of chirality in different dimensions are brought together and embedded in one molecular system, a chirality hierarchy will then be generated. For example, in helical structures, which is of 1D chirality, of proteins and DNA, amino acid residues or deoxyribose sugars contain chiral centers (OD chirality), so proteins and DNA structures form a chirality hierarchy with two kinds of chirality, OD and 1D. In principle, it is possible that a 3D chiral supramolecular structure contains planar (2D) chirality, whose structure also possesses axial (1D) chirality and (OD) chiral centers, thus forming a complete set of chirality hierarchy.

Very recently, based on the model results for proteins and DNA to attribute the origin of homochirality, we proposed the Principle of Chirality Hierarchy, ${ }^{5}$ which states that the propensity of lower dimension chirality is dictated by that of the higher dimension chirality. One consequence of this Principle is homochirality, as well shown in proteins and DNA, a striking feature of these life-essential biomacromolecules with multiple chiral centers, ${ }^{8-10}$ where all chiral centers in a system possess the same chirality. We have shown that righthanded $\alpha$-helix and $3_{10}$-helix structures in proteins both favor the L-chiral form of amino acids, 
whereas deoxyribose sugars in right-handed DNA helical structures prefer the D-chiral form instead. ${ }^{5}$ We also identified the existence of strong cooperativity effect, ${ }^{5-7}$ which is dominated by the favorable electrostatic interaction in the homochiral conformation through the analysis of total energy partition ${ }^{11,12}$ and information-theoretic approach. ${ }^{13,14}$

In this work, we confirm the validity of the Principle of Chirality Hierarchy in an another axial chirality system, a left-handed three-blade propeller molecule (Scheme 1). There are three components to make a propeller molecule in Scheme 1, including the propeller central atom $X$, the six-membered-ring $(6 M R)$, and the number and location of chiral groups on the $6 \mathrm{MR}$, which can be at either the meta position, or para position, or both. The purpose of the present study is to answer the following three questions. (i) When one or more chiral groups are placed on the $6 \mathrm{MR}$ of the propeller blade, will there be any stability difference between its $\mathrm{R}$ and $\mathrm{S}$ stereoisomers? (ii) What is the impact of the three components of the blade on the $\mathrm{R} / \mathrm{S}$ stability difference? In other words, how does the higher hierarchical chirality influence the property of the lower hierarchical chirality? (iii) Is the feature of homochirality still valid for this model system? If yes, what is the role of the electrostatic interaction dominance and cooperativity effect?

To that end, as shown in Scheme 1, we build the three-blade propeller model with 6 options for the center $\mathrm{X}, \mathrm{X}=\mathrm{Al}, \mathrm{B}, \mathrm{C}^{+}, \mathrm{C}^{-}, \mathrm{N}$ and $\mathrm{P}, 8$ alternatives for the six-membered ring, $6 \mathrm{MR}=$ phenyl, pyridine, pyrimidine, pyrazine, triazine, and borazine, and 10 choices for the chiral groups, $C^{*} R_{1} R_{2} R_{3}$ with $R_{1} R_{2} R_{3}=H, F, C l, O H$, and $M e$, in either $R$ or $S$ conformer attached to the meta, para, or both positions. Blade angles defined as the dihedral angle between two blades for one of the systems are available as an illustrative example in SI Table S1. To examine the homochirality propensity and interaction dominance, we choose one model with 6 chiral centers and consider all possible chirality permutations, yielding $2^{6}=64$ stereoisomers. To analyze the cooperativity effect, ${ }^{5,7,15-17}$ we gradually add chiral groups to the $6 \mathrm{MR}$, and observe the $S$ - $R$ stereoisomeric energetic difference to quantify the enhancement of the energetic 
difference as more chiral groups are added to the 6MR of the blades.

Table 1 shows the dependence of the stereoisomeric energy difference (S-R) on basis sets and methodologies for three models, where with $X=B, 6 M R=$ phenyl, the chiral group is CHFMe, and chiral groups are placed on meta, para, and two meta positions of each blade, respectively. Results in the last row with the CBSB7 basis set and MP2 method can be regarded as the reference. As an illustration, Figure 1 exhibits the optimized structure of $R$ and $S$ conformers of the model at the MP2/6-311G(d) level of theory with chiral groups all at the meta position. As can be seen from the Table, (i) the stereoisomeric energy difference is always no zero, suggesting that when chiral centers are placed on 6MRs, there will always be a stability difference between $R$ and $S$ configurations, yielding a thermodynamic preference of one conformer over the other. (ii) This stability difference depends on where and how many these chiral centers are placed. (iii) Compared with the results from the complete basis set and MP2 method, the stereoisomeric energy difference does not significantly depend on the choice of basis sets and approximate density functionals. It is well known that there is no energetic difference between a pair of enantiomers, $R$ and $S$. For example, the $R$ configuration of CHFMe (OD, point chirality) should have the same energy as its mirror image, the $S$ configuration. However, when it is put on blades of a propeller molecule (with 1D, axial chirality) in Scheme 1, such as the one shown in Fig. 1, which henceforth forms a 0D-1D chirality hierarchy, discernible differences in energy from the two stereoisomers are yielded. The results in Table 1 numerically confirm this point. They also show that chiral centers placed at different positions and with different numbers on the blade could generate substantially different results, highlighting the validity of the Principle of Chirality Hierarchy.

This stereoisomeric energy difference also depends on the nature of the three components of the blade. Table 2 displays the result for the impact of the propeller center, where six choices, $\mathrm{Al}, \mathrm{B}, \mathrm{C}^{+}, \mathrm{C}^{-}, \mathrm{N}$ and $\mathrm{P}$, were examined for ten chiral groups located on only one meta position of the three blades. It is seen from the Table that the $S-R$ stereoisomeric 
energy difference can be both negative and positive with the same propeller center but with different chiral groups. The same is true for the same chiral group but different propeller centers. This result confirms that the nature of the axial chirality (i.e., the 3-blade propeller) determines the propensity of the point chirality (i.e., the preference of $R / S$ configurations and the stability difference for the chiral group).

In Table 3, we studied the impact of the 6MR on the stereoisomeric energy difference. In the study, we chose 8 options of $6 \mathrm{MR}$ as the blade of the propeller (Scheme 1). Again, as shown in the Table, the $S-R$ stereoisomeric energy difference can be both positive and negative except for two cases, when Al is the propeller center, and pyridine and pyrimidine are the blade. In these two cases, we found that the two stereoisomers become mirror images to each other (since blade angles are exceedingly small and one propeller switches from lefthandedness to right-handedness), so their stereoisomeric energy values become identical. In the same spirit, in Table 4, we examined the impact of the number and position of chiral groups on 6MRs on the stereoisomeric energy difference, where we considered five possible ways to place chiral groups on 6MRs. Again, same as Table 3, the S-R energy difference can be both negative and positive with the same chiral group at different locations, or at the same location but with different chiral groups. Put together, it is adequate to conclude that it is the axial chirality of the propeller that dictates the stereoisomeric energetic preference of the point chirality on 6MRs.

To find out if cooperativity effect ${ }^{5,7,15-17}$ is still present in this propeller system, we chose one model from Scheme 1 ( $\mathrm{B}$ as the propeller center and phenyl as the blade) and then gradually increased the number of chiral groups at the meta positions of the blades from 1 to 6 with either $R$ or $S$ configuration. After six groups are all added, the all- $R$ and all-S conformers become the two structures in Fig. 1. Figure 2 shows (a) the strong linear relationship between the stereoisomeric energy difference and the number of chiral groups and (b) the cooperativity profile. From the cooperativity profile in Fig. $2 b$, whose tendency is going 
downwards, according to the definition of cooperativity, ${ }^{5}$ we know that there exists positive cooperativity in this system. This means that when more chiral groups are added to the blade, the average stereoisomeric energy difference will become even larger in magnitude. Therefore, this is an enhancement effect to make the energy gap between all- $R$ and all-S stereoisomers more significant as more chiral groups are added to the blade.

What is the driving force behind? To answer this question, based on the structures in Fig. 1 , we built $2^{6}=64$ heterochiral models by permutating all 6 chiral centers on the blades with either $R$ or $S$ configuration. All models we considered thus far are homochiral. These 64 models built by permutation include 62 heterochiral structures, plus two homochiral ones, one all $R$ and the other all $S$. With these 64 models, we can answer the question if homochirality is a feature of this propeller system. Details of the total energy and its components for the 64 stereoisomers are available in SI Table S2. From the Table, we can see that the all-S homochiral isomer possesses the lowest energy, so it is the most stable isomer. This result confirms that homochirality is indeed a remarkable feature of this propeller system, same as the helical structures reported in the literature. ${ }^{5,7}$

Included in Fig. 3 are energetic analysis results for the 64 stereoisomers. In Fig. 3a, a strong linear correlation between the stereoisomeric stability (with all- $R$ isomer as the reference) and the electrostatic energy component is obtained, suggesting that (i) the electrostatic interaction in the all-S form is stronger than that in any other isomers and (ii) it is the electrostatic component that dominates the energetic contribution to the stereoisomeric stability. This result is consistent with that reported previously for other systems. ${ }^{18-25}$ In Figs. $3 b$ and $3 c$, when the electrostatic component is fitted together with either the exchangecorrelation or steric energy, better correlations can be obtained, suggesting that effects from exchange-correlation and steric components play minor yet indispensable roles., ${ }^{78-25}$

Besides the energetic analysis, the stability preference of a chirality hierarchy can also be appreciated by quantities associated with the electron density, such as simple density 
functionals from the information-theoretic approach (ITA) in density functional theory. ${ }^{13,14}$ Shown in Fig. 4 are a few strong correlations of the stereoisomer stability with ITA quantities, such as Fisher information, ${ }^{26}$ Ghosh-Berkowitz-Parr entropy, relative Fisher information, ${ }^{27-29}$ etc. These strong correlations demonstrate that besides energetic differences, chirality propensity and stability property can additionally be reflected in the electron density distribution. For example, Fisher information gauges the electron density heterogeneity and steric effect, ${ }^{11,30}$ so a strong correlation with this quantity is an indication that heterochiral models possess stronger electron density heterogeneity and larger steric repulsion due to the electron density redistribution from the chirality rearrangement. The same analysis could be applied to other ITA quantities in Fig. 4.

In summary, in this work, the validity of Principle of Chirality Hierarchy has been verified by the three-blade propeller molecular system. Two prominent features are unambiguously demonstrated. At first, for this OD-1D chirality hierarchy system, the chirality preference of the OD (point) chirality is determined by the 1D axial chirality of the left-handed propeller. Secondly, homochirality is favored over heterochirality. We also observed the existence of positive cooperativity in this system with the electrostatic interaction playing the dominant role while the roles of the exchange-correlation and steric effects are minor yet indispensable. These results, together with the ones from our previous studies for helical structures, should pave the pathway to make this principle generally applicable to studies in asymmetric synthesis, macromolecular assembly, and many others.

Supporting Information Available: Terminology and model building methods, computational details, blade angles of 10 chiral groups at the meta position with 6 centers, and energetic decomposition results for 64 heterochiral models, together with the Cartesian coordinate of all the optimized structures discussed in this work are available free of charge via the Internet at http://pubs.acs.org. 


\section{References}

1. McMurry, J.; Simanek, E. Fundamentals of Organic Chemistry (Brooks Cole, ed. 6, 2006).

2. Moss, G.P. Basic terminology of stereochemistry, Pure \& Appl. Chem. 1996, 68, 2193 2222.

3. Elemans, J.A.A.W.; Cat, I.D.; Xu, H.; Feyter, S.D. Two-Dimensional Chirality at Liquid-Solid Interfaces, Chem. Soc. Rev. 2009, 38, 722-736.

4. Randić, M. Graph Theoretical Descriptors of Two-Dimensional Chirality with Possible Extension to Three-Dimensional Chirality, J. Chem. Inf. Comput. Sci. 2001, 41, 639-649.

5. Liu, S.B. Homochirality originates from handedness of helices. J. Phys. Chem. Lett. 2020, $11,8690-8696$.

6. Xin, X. Thermodynamic Origin of Homochirality for Macromolecules in Nature. Acta Phys.Chim. Sin. 2021, 37, 2011078.

7. Li, M; He, X.; Chen, J.; Wang, B.; Liu, S.B.; Rong, C. Density functional theory and information-theoretic approach study on the origin of homochirality in helix structures. $J$. Phys. Chem. A 2021, 125, $1269-1278$.

8. Hein, J.E.; Blackmond, D.G. On the Origin of Single Chirality of Amino Acids and Sugars in Biogenesis. Acc. Chem. Res. 2012, 45, 2045-2054.

9. Skolnick, J.; Zhou, H.; Gao, M. On the Possible Origin of Protein Homochirality, Structure, and Biochemical Function, Proc. Natl. Acad. Sci. 2019, 116, 26571-26579.

10. Blackmond, D.G. Autocatalytic Models for the Origin of Biological Homochirality. Chem. Rev. 2020, 11, 4831 - 4847.

11. Liu, S.B. Steric effect: A Quantitative Description from Density Functional Theory. J. Chem. Phys. 2007, 126, 244103.

12. Liu, S.B. Conceptual Density Functional Theory and Some Recent Developments. Acta Phys.-Chim. Sin. 2009, 25, 590-600.

13. Liu, S.B. Information-theoretic approach in density functional reactivity theory. Acta Phys.-Chim. Sin. 2016, 32, 98-118.

14. Rong, C.; Wang, B.; Zhao, D.; Liu, S.B. Information-theoretic approach in density functional theory and its recent applications to chemical problems. Wiley Interdiscip. Rev.: Comput. Mol. Sci. 2020, 10, e1461.

15. Rong, C.; Zhao, D.; Yu, D.; Liu, S.B. Quantification and Origin of Cooperativity: Insights from Density Functional Reactivity Theory. Phys. Chem. Chem. Phys. 2018, 20, 17990- 17998.

16. Zhou, T.; Liu, S.; Yu, D.; Zhao, D.; Rong, C.; Liu, S.B. On the Negative Cooperativity of Argon Clusters Containing One Lithium Cation or Fluorine Anion. Chem. Phys. Lett. 2019, 716, 
$192-198$.

17. Rong, C.; Zhao, D.; Zhou, T.; Liu, S.; Yu, D.; Liu, S.B. Homogeneous Molecular Systems Are Positively Cooperative but Charged Molecular Systems Are Negatively Cooperative. J. Phys. Chem. Lett. 2019, 10, 1716- 1721.

18. Liu, S.B. Origin and Nature of Bond Rotation Barriers: A Unified View, J. Phys. Chem. A 2013, 17, 962-965.

19. Liu, S.B.; Hu, H.; Pedersen, L.G. Steric, Quantum, and Electrostatic Effects on SN2 Reaction Barriers in Gas Phase, J. Phys. Chem. A 2010, 114, 5913-5918.

20. Ess, D. H.; Liu, S.B.; De Proft, F. Density Functional Steric Analysis of Linear and Branched Alkanes, J. Phys. Chem. A 2010, 114, 12952-12957.

21. Huang, Y.; Zhong, A.; Yang, Q; Liu, S.B. Origin of Anomeric Effect: A Density Functional Steric Analysis, J. Chem. Phys. 2011, 134, 084103.

22. Liu, S.B.; Schauer, C.K. Origin of Molecular Conformational Stability: Perspectives from Molecular Orbital Interactions and Density Functional Reactivity Theory, J. Chem. Phys. 2015, 142, 054107.

23. Cao, X.; Liu, S.; Rong, C.; Lu, T.; Liu, S.B. Is There a Generalized Anomeric Effect? Analyses from Energy Components and Information-Theoretic Quantities from Density Functional Reactivity Theory, Chem. Phys. Lett. 2017, 687, $131-137$.

24. Zhao, D.; Liu, S.B.; Rong, C.; Zhong, A.; Liu, S.B. Towards Understanding Isomeric Stability of Fullerenes with Density Functional Theory and Information-Theoretic Approach, ACS Omega, 2018, 3, 17986-17990.

25. Wang, B.; Yu, D.; Zhao, D.; Rong, C.; Liu, S.B. Nature and Origin of $\psi$-Gauche Effect in Sulfoxides: A Density Functional Theory and Information-Theoretic Approach Study, Chem. Phys. Lett. 2019, 730, $451-459$.

26. Fisher, R. A. Theory of Statistical Estimation. Math. Proc. Cambridge Philos. Soc. 1925, 22, $700-725$.

27. Ghosh, S. K.; Berkowitz, M.; Parr, R. G. Transcription of Ground-State Density-Functional Theory into a Local Thermodynamics. Proc. Natl. Acad. Sci. U.S.A. 1984, 81, 8028- 8031.

28. Liu, S.B. Identity for Kullback-Leibler Divergence in Density Functional Reactivity Theory. J. Chem. Phys. 2019, 151, 141103.

29. Wang, B.; Zhao, D.; Lu, T.; Liu, S.B.; Rong, C. Quantifications and applications of relative Fisher information in density functional theory. J. Phys. Chem. A 2021, 125, $3802-3811$.

30. Liu, S.B. On the relationship between densities of Shannon entropy and Fisher information for atoms and molecules. J. Chem. Phys. 2007, 126, 191107. 
Table 1. Benchmark results of the stereoisomeric energy difference $(S-R)$ with eight basis sets and seven approximate density functionals for three models in Scheme 1 with the chiral centers at meta, para, and two meta positions on each six-membered ring. The propeller center is $\mathrm{B}$ atom and the chiral group is carbon linked with $\mathrm{H}, \mathrm{Me}$, and $\mathrm{F}$ in both $R$ and $S$ configurations. In the Meta case, the chiral group is at a meta position of the six-membered ring, whereas in the Para case, it is at the para position. For the Meta/Meta case, both meta positions were taken by the chiral group. The CBSB and MP2 result can be served as the reference in basis set and approximate functional tests, respectively. In basis set benchmarks, wB97XD was used, and in methodology tests, 6-311G(d) was employed. Units in $\mathrm{kcal} / \mathrm{mol}$.

\begin{tabular}{lrrrlrrr}
\hline Basis Set & Meta/Meta & Meta & Para & Functional & Meta/Meta & Meta & Para \\
\hline $6-31 G(d)$ & -4.16 & -0.42 & -0.09 & B3LYP & -3.32 & 0.11 & -0.08 \\
6-311G(d) & -4.09 & -0.42 & -0.10 & PBEPBE & -3.83 & -0.02 & -0.08 \\
6-311+G(d) & -3.45 & -0.08 & -0.12 & HCTH & -3.71 & -0.09 & -0.06 \\
6-311++G(d,p) & -3.45 & -0.10 & -0.13 & TPSSTPSS & -3.46 & 0.04 & -0.09 \\
Def2TZVP & -3.15 & -0.17 & -0.10 & WB97XD & -4.09 & -0.42 & -0.10 \\
DGDZVP & -3.43 & -0.25 & -0.12 & M062X & -4.02 & -0.46 & -0.09 \\
cc-pVDZ & -4.25 & -0.43 & -0.08 & APFD & -4.57 & -0.38 & -0.10 \\
CBSB7 & -4.08 & -0.41 & -0.10 & MP2 & -4.32 & -0.37 & -0.08 \\
\hline
\end{tabular}


Table 2. The dependences of the stereoisomeric energy difference $(S-R)$ on the $X$ center in Scheme 1. A total of six centers, $\mathrm{Al}, \mathrm{B}, \mathrm{C}^{+}, \mathrm{C}^{-}, \mathrm{N}$ and $\mathrm{P}$, were examined for the model system with $\mathrm{B}=\mathrm{C}=$ hydrogen atom, and $\mathrm{A}$ takes one of the ten chiral groups in both $R$ and $S$ configurations. Units in $\mathrm{kcal} / \mathrm{mol}$.

\begin{tabular}{lrrrrrr}
\hline $\mathbf{R}_{\mathbf{1}} \mathbf{R}_{\mathbf{2}} \mathbf{R} \mathbf{R}_{\mathbf{3}}$ & Al & $\mathbf{B}$ & $\mathbf{C}^{+}$ & $\mathbf{C}^{-}$ & $\mathbf{N}$ & $\mathbf{P}$ \\
\hline H_F_Cl & -0.40 & -0.37 & -1.15 & -0.34 & -0.74 & 1.31 \\
H_Me_Cl & 0.36 & 0.38 & 0.99 & -0.16 & 0.22 & -1.61 \\
H_Me_F & -0.04 & -0.16 & -0.12 & -0.53 & -0.69 & 0.23 \\
H_Me_OH & -0.33 & -0.62 & -0.58 & -1.15 & -1.04 & 0.38 \\
H_OH_Cl & -0.68 & -0.62 & -1.93 & -0.38 & -0.88 & -0.75 \\
H_OH_F & -0.79 & -0.16 & 0.03 & -0.03 & -0.75 & -1.23 \\
Me_F_Cl & -0.82 & -1.13 & 1.67 & -2.73 & -0.70 & -0.76 \\
Me_OH_Cl & -1.19 & -1.04 & -2.23 & 0.17 & -0.94 & -0.89 \\
Me_OH_F & -0.15 & -0.27 & -0.13 & 0.01 & -0.01 & -0.07 \\
OH_F_Cl & -0.05 & 0.11 & -0.11 & 0.23 & 0.44 & 0.05 \\
\hline
\end{tabular}


Table 3. The impact of the six-membered ring on the stereoisomeric energy difference $(S-R)$ in Scheme 1 for six $X$ centers with the chiral group of $B=$ hydrogen atom and $A=C=C H M e F$ at the meta position in both $R$ and $S$ configurations. Units in $\mathrm{kcal} / \mathrm{mol}$.

\begin{tabular}{crrrrrr}
\hline 6MR Group & Al & B & $\mathbf{C}^{+}$ & $\mathbf{C}^{-}$ & $\mathbf{N}$ & $\mathbf{P}$ \\
\hline 1 & -3.16 & -4.09 & -4.01 & -4.61 & -3.67 & -1.52 \\
2 & -3.21 & -4.40 & -5.37 & -4.86 & -5.51 & -5.89 \\
3 & 0.00 & -2.27 & -1.72 & -2.25 & -2.76 & -2.95 \\
4 & -2.27 & -3.49 & -4.38 & -5.17 & -5.73 & -2.72 \\
5 & -0.30 & -0.44 & -1.10 & -1.86 & -2.49 & -3.95 \\
6 & -1.02 & -2.85 & -2.61 & -3.20 & -3.15 & 0.40 \\
7 & 0.00 & -1.20 & -0.84 & -2.92 & -2.65 & -1.88 \\
8 & 0.90 & 0.95 & 0.68 & 2.24 & 3.77 & 0.14 \\
\hline
\end{tabular}


Table 4. The influence of the number and position of chiral groups on each six-membered ring to the stereoisomeric energy difference $(S-R)$ in Scheme 1 for ten chiral groups between their both $R$ and $S$ states. In the Meta case, $\mathrm{A}$ is one of the ten groups, and $\mathrm{B}=\mathrm{C}=$ hydrogen atom. In the Para case, $A=C=$ hydrogen, and $B$ takes one of the ten chiral groups. In the Meta/Meta case, $B=H$, and $A=C$. In the Meta/Para case, $A=B$ and $C=H$. In the Meta/Para/Meta case, $\mathrm{A}=\mathrm{B}=\mathrm{C}$. Units in $\mathrm{kcal} / \mathrm{mol}$.

\begin{tabular}{lrrrrr}
\hline R $_{\mathbf{1}} \mathbf{R}_{\mathbf{2}} \mathbf{R}_{\mathbf{3}}$ & Meta & Para & Meta/Meta & Meta/Para & Meta/Para/Meta \\
\hline H_F_Cl & -0.37 & -0.01 & 1.58 & 0.04 & 1.93 \\
H_Me_Cl & 0.38 & -0.16 & -2.43 & -0.20 & -4.31 \\
H_Me_F & -0.16 & -0.14 & -4.09 & -1.00 & -6.15 \\
H_Me_OH & -0.62 & -0.14 & -4.95 & -1.23 & -7.06 \\
H_OH_Cl & -0.62 & 0.01 & 1.68 & 0.47 & 3.26 \\
H_OH_F & -0.16 & 0.43 & -1.10 & 0.18 & 0.42 \\
Me_F_Cl & -1.13 & 0.02 & 2.67 & 2.63 & -0.07 \\
Me_OH_Cl & -1.04 & 0.02 & 1.26 & -2.90 & 5.32 \\
Me_OH_F & -0.27 & -0.05 & -0.97 & -0.99 & -6.27 \\
OH_F_Cl & 0.11 & -0.01 & -0.43 & 0.37 & -1.89 \\
\hline
\end{tabular}




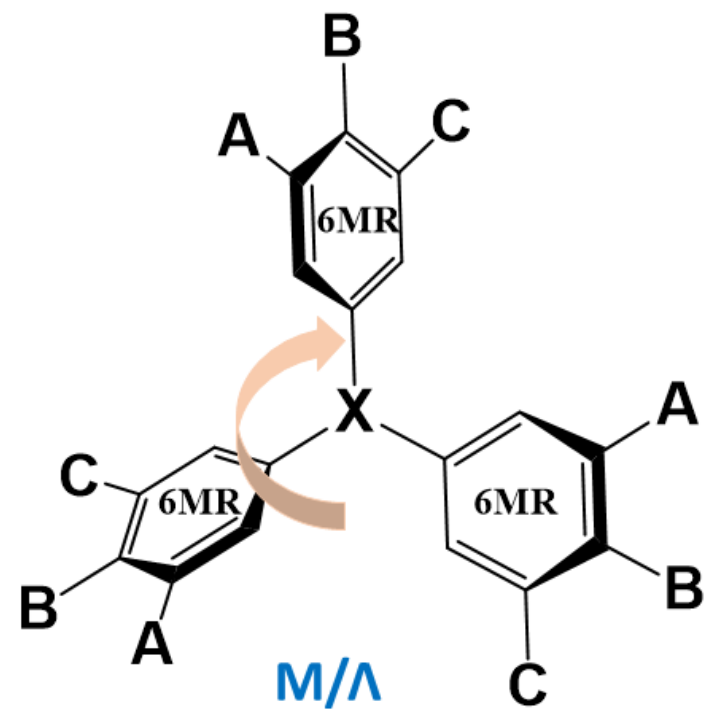

$X=A l, B, C^{+}, C^{-}, N, P$

$A, B, C=C^{*} R_{1} R_{2} R_{3}(R$ or $S)$

$\mathrm{R}_{1} \mathrm{R}_{2} \mathrm{R}_{\mathbf{3}}=\mathrm{H}, \mathrm{F}, \mathrm{Cl}, \mathrm{OH}, \mathrm{Me}$

$6 \mathrm{MR}$

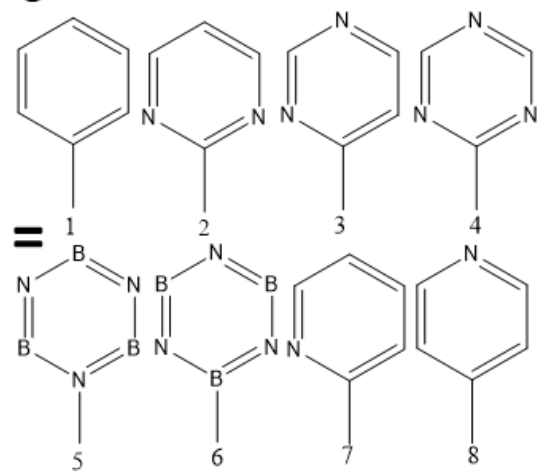

Scheme 1. Propeller molecular models as a chirality hierarchy studied in this work. The lefthanded [clockwise, $\mathrm{M}$ (minus) or $\Lambda$ ] three-blade propeller molecular system with 6 options for the center $\mathrm{X}, 8$ alternatives for the six-membered ring, and 10 choices for the chiral groups attached to the meta and/or para positions in both $R$ and $S$ configurations. 


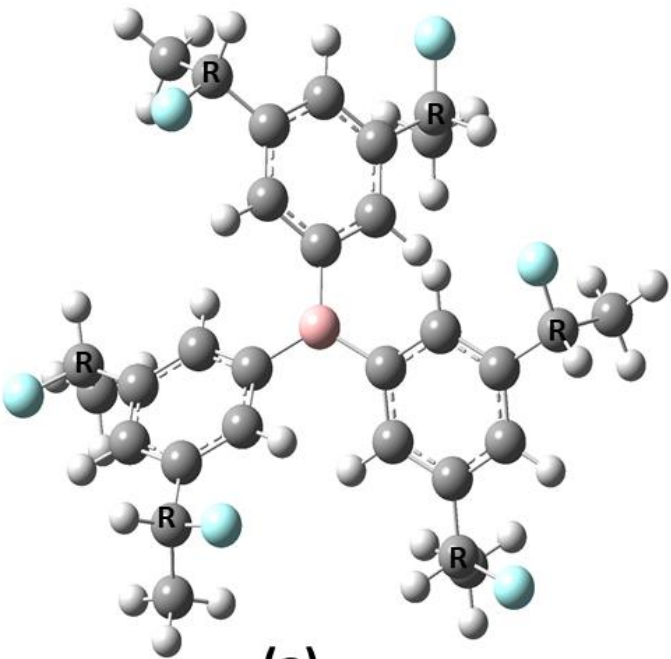

(a)

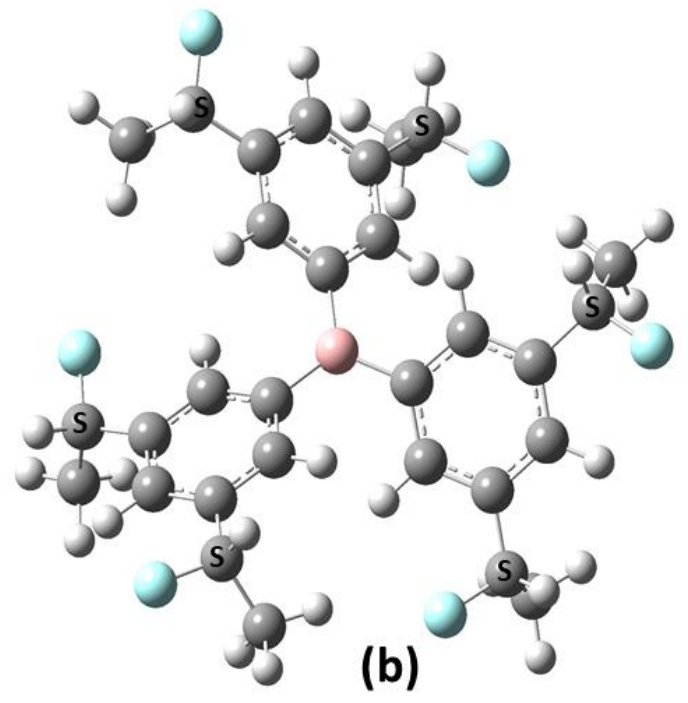

(b)

Figure 1. The optimized structure of (a) all-R and (b) all-S conformers of the three-blade model at the MP2/6-311G(d) level of theory with $\mathrm{X}=\mathrm{B}, 6 \mathrm{MR}=$ phenyl, and the chiral group $\mathrm{C}^{*} \mathrm{HFMe}$ at all meta positions. The blade angle is $53.2^{\circ}$ and $49.6^{\circ}$ for all- $R$ and all-S conformers, respectively. 

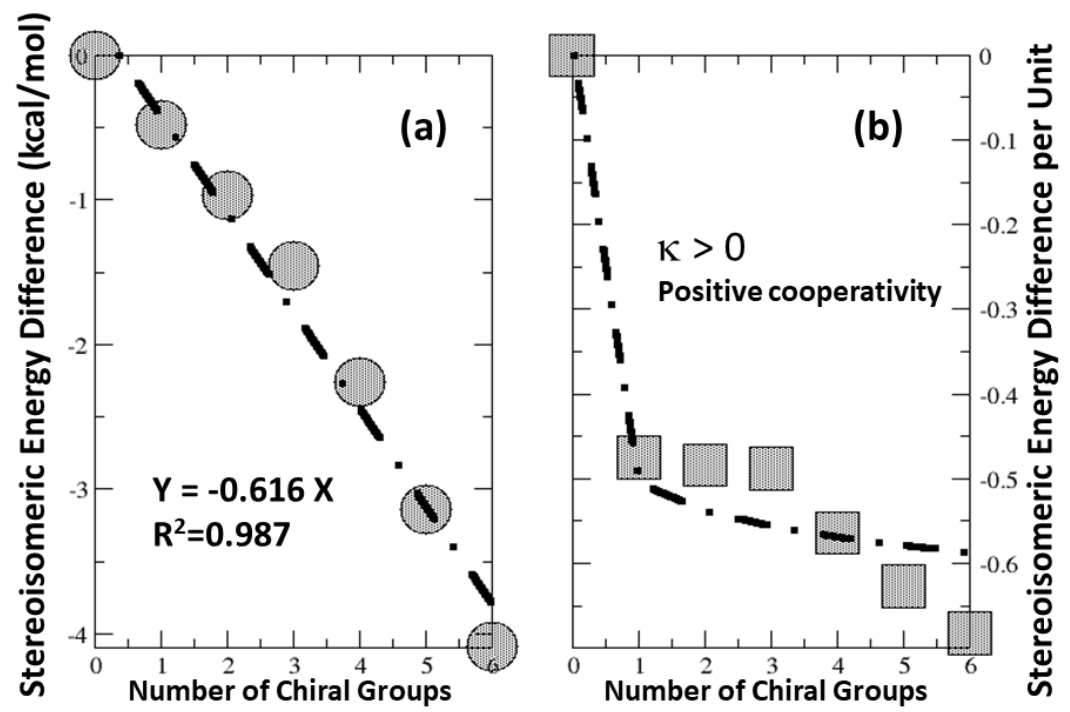

Figure 2. (a) The relationship between the stereoisomeric energy difference and the number of chiral groups and (b) the cooperativity profile (the stereoisomeric energy difference per chiral group vs. the number of chiral groups) for the model system in Figure 1. For Numbers $1-3$, each blade is consecutively added one chiral group, and for Numbers $4-6$, each blade is added another chiral group. 

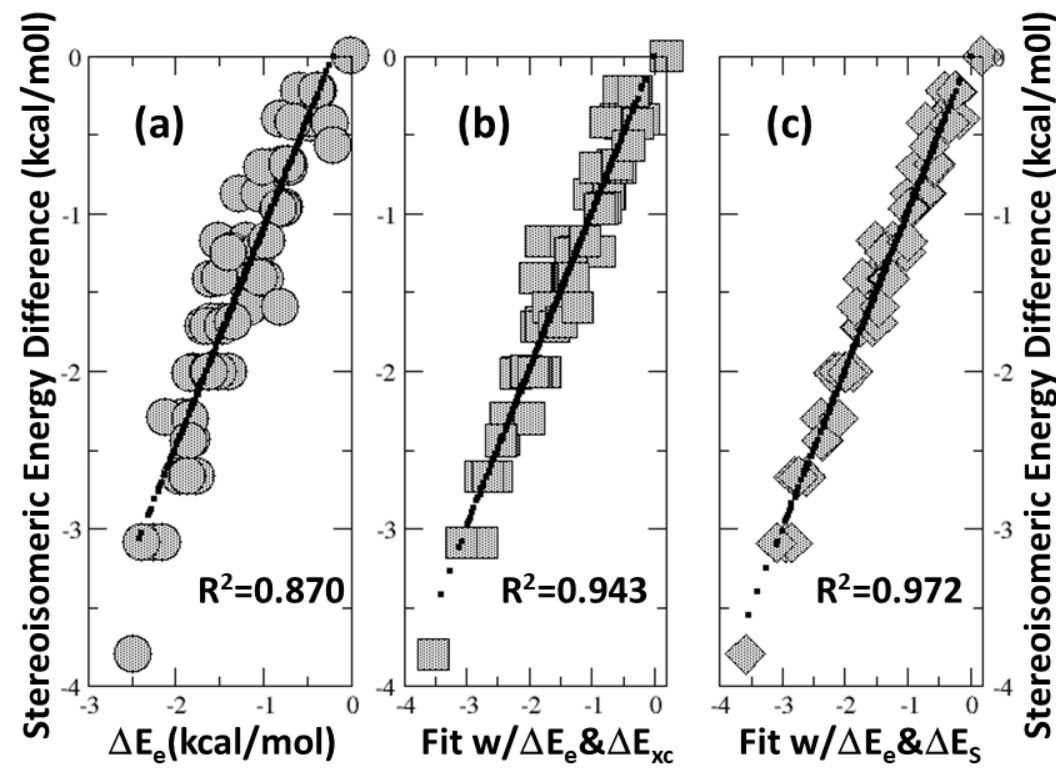

Figure 3. Energetic analyses for 64 heterochiral isomers in Figure 1. (a) The strong linear correlation of the stereoisomeric energy difference (with all-R as the reference) with (a) the electrostatic component; (b) two-variable fitting with electrostatic and exchange-correlation energy components; and (c) two-variable fitting with electrostatic and steric components. 


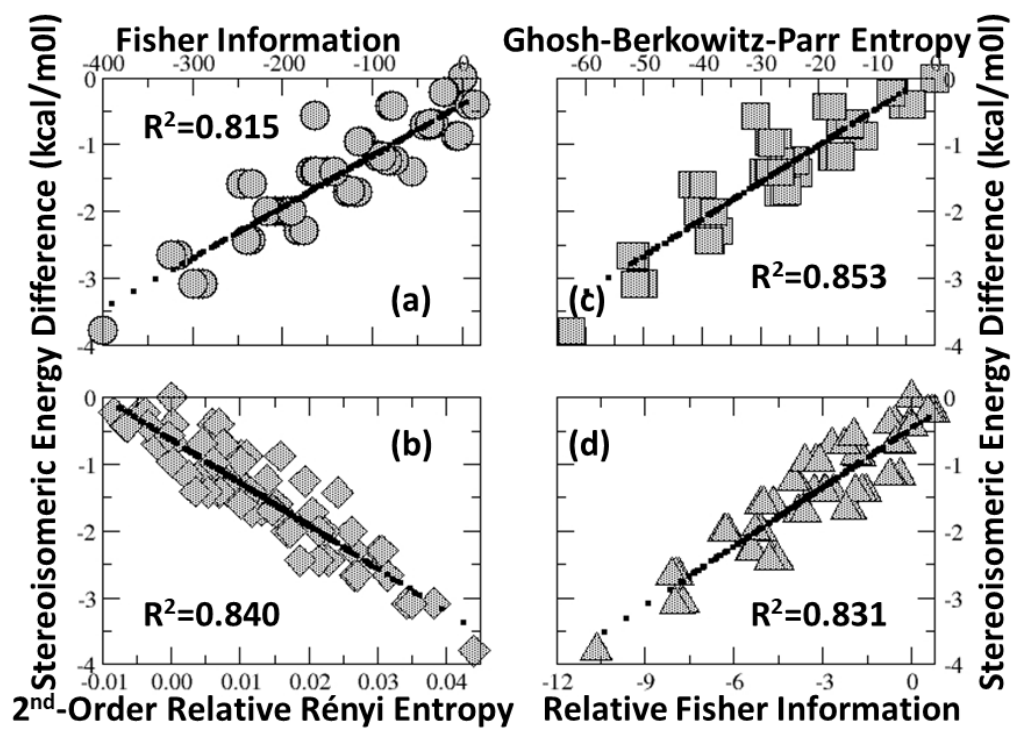

Figure 4. Strong linear correlations of the stereoisomeric energy difference (with all-R homochiral isomer as the reference) with four ITA quantities, Fisher information, GhoshBerkowitz-Parr entropy, $2^{\text {nd }}$-order Rényi entropy, and relative Fisher information for the 64 heterochiral modes based on structures in Figure 1. 